\title{
ENTWICKLUNG GEWISSER DIELEKTRISCHER GRUNDLÖSUNGEN IN ORTHONORMALREIHEN
}

\author{
REINER KÜHNAU
}

\section{Einleitung}

Die komplexe Differentialgleichung

$$
f_{\bar{z}}=v \overline{f_{z}}, \quad v=v(z), \quad|v| \leqq v_{0} \ll 1, \quad z=x+i y,
$$

für eine gesuchte Funktion $f=f(z)$ tritt bekanntlich in vielen Situationen der mathematischen Physik (z. B. Elektrostatik, Strömungen in porösen Medien im ebenen Fall) als auch in der Theorie der Extremalprobleme bei quasikonformen Abbildungen mit ortsabhängiger Dilatationsbeschränkung auf (vgl. z. B. die Literatur in [1], [7]). In [7] wurde ein Verfahren entwickelt, das über Orthonormalreihenentwicklungen von analytischen Funktionen ein Berechnungsverfahren für Grundlösungen vorgegebenen Singularitätentypus liefert im Falle, es ist $v(z) \equiv 0$ in einem Gebiet $\mathfrak{b}$ und $v(z) \equiv q \quad(0<q<1)$ im Komplement. Im folgenden soll gezeigt werden, wie sich dieses Verfahren bis hin zur wirklichen numerischen Durchführung und Fehlerabschätzung besonders günstig gestaltet bei Verwendung eines speziellen Orthonormalsystems, das aus der Riemannschen Abbildungsfunktion heraus entwickelt wird.

Es sei hinfort $\mathfrak{5}$ ein $z=\infty$ im Innern enthaltendes Gebiet, welches von einer einzigen Jordankurve $\mathfrak{C}$ (geschlossen und so orientiert, dass $\mathfrak{G}$ zur Linken liegt) berandet wird, die sich aus endlich vielen abgeschlossenen analytischen Bögen zusammensetzt, die unter von 0 verschiedenem Winkel zusammenstoßen. Wir können o.E.d.A. bei $\mathfrak{G}$ den konformen Radius 1 annehmen. Die anzuwendende Berechnungsprozedur besteht nach [7] kurz im folgenden.

Es sei $\mathfrak{H}$ die Gesamtheit der $F^{\prime}(z) \in L^{2}(\mathfrak{5})$, für die $F(z)$ in $\mathfrak{G}$ regulär ist mit $F(\infty)=0$. Jedem $F^{\prime}(z) \in \mathfrak{H}$ sei eine in $\mathfrak{G}$ ebenfalls reguläre Funktion $F^{*}(z)$ zugeordnet durch eine Vorschrift, die sich für noch auf $\mathfrak{C}$ hölderstetige $F$ so schreibt:

$$
F^{*}(z)=\frac{q}{2 \pi i} \int_{\mathfrak{c}} \frac{\overline{F(t)}}{t-z} d t .
$$

Es ist dann $F^{*}(\infty)=0$, und $q \bar{F}-F^{*}$ ist ins Innere von $\mathbb{C}$ analytisch fortsetzbar, insbesondere $F^{*}$ noch auf $\mathfrak{C}$ stetig, und diese Eigenschaft charakterisiert $F^{*} . \mathfrak{H}$ wird zu einem separablen Hilbertraum mit reproduzierender Kernfunktion $K(z, t)$ durch ein Skalarprodukt, welches sich bei Randregularität von $F^{\prime}, G^{\prime} \in \mathfrak{H}$ (sonst 
wird statt $\mathfrak{C}$ eine Niveaulinie genommen, die hernach nach $\mathfrak{C}$ strebt) so schreibt:

$$
\left(F^{\prime}, G^{\prime}\right)=-\frac{1}{2 i} \int_{\mathfrak{C}} F \overline{d G}+\overline{F^{*}} d G^{*}
$$

Mit der hierzu gehörigen Norm $\|\ldots\|$ gilt

$$
\left(1-q^{2}\right) \iint_{\mathfrak{G}}\left|F^{\prime}\right|^{2} d x d y \leqq\left\|F^{\prime}\right\|^{2} \leqq \iint_{\mathfrak{G}}\left|F^{\prime}\right|^{2} d x d y .
$$

Bei Weglassung des (für unsere Ziele hier gerade entscheidenden) zweiten Anteils im Integranden von (3) entsteht der klassische Hilbertraum $\mathfrak{H}^{\prime}$ der quadratisch integrierbaren $F^{\prime}$ mit der Bergmanschen Kernfunktion. Jedes vollständige Funktionensystem von $\mathfrak{H}^{\prime}$ ist ein solches von $\mathfrak{H}$ (und umgekehrt), und wir können die allgemeine Theorie der Kernfunktion (vgl. z. B. [12]) anwenden.

\section{Darstellungen für Grundlösungen}

Uns geht es nun wesentlich um als Grundlösungen deutbare quasikonforme Normalabbildungen. Wir wollen uns auf folgende Aufgabe beschränken, die unmittelbar Verallgemeinerungsmöglichkeiten erkennen läßt. Wir wollen nämlich diejenigen Funktionen $g_{\vartheta}(z)$ explizit bestimmen, die für alle endlichen $z$ stetig sind, dabei in $\mathfrak{G}$ regulär bis auf einen Pol erster Ordnung in $z=\infty$ mit der hydrodynamischen Normierung

$$
g_{\vartheta}(z)=z+\frac{A_{1, \vartheta}}{z}+\ldots,
$$

und für die $g_{\vartheta}(z)-e^{2 i \vartheta} q \frac{z}{g_{\vartheta}(z)}$ im Innern von $\mathfrak{C}$ regulär ist, so daß $e^{-i \vartheta} g_{\vartheta}(z)$ schlicht ist und (1) mit dem angegebenen speziellen $v$ erfüllt. Wegen der Schlichtheit ist $g_{\vartheta}^{\prime}-1 \in \mathfrak{S}^{\prime}$. Wir bilden

$$
M(z)=\frac{1}{2}\left[g_{0}(z)-g_{\pi / 2}(z)\right], \quad N(z)=\frac{1}{2}\left[g_{0}(z)+g_{\pi / 2}(z)\right] .
$$

Dann gilt nach [7] (dort nach Grenzübergang $\zeta \rightarrow \infty$ ) oder [3] mit einem im Sinne unseres Skalarproduktes vollständigen Orthonormalsystem $\left\{\varphi_{k}(z)\right\}$

$$
M^{\prime}(z)=\sum_{k}\left(M^{\prime}, \varphi_{k}^{\prime}\right) \varphi_{k}^{\prime}(z), \quad N^{\prime}(z)-1=\sum_{k}\left(N^{\prime}-1, \varphi_{k}^{\prime}\right) \varphi_{k}^{\prime}(z) .
$$

Dabei lassen sich - das ist der ,Clou“ der ganzen Prozedur — die hier in Form von Skalarprodukten auftretenden Koeffizienten $\alpha_{k}, \beta_{k}$ wirklich bestimmen für in $\overline{\mathfrak{G}}$ noch hölderstetige $\varphi_{k}$ :

$$
\begin{gathered}
\alpha_{k}=\left(M^{\prime}, \varphi_{k}^{\prime}\right)=\frac{1}{2 i} \int_{\mathfrak{C}}\left[q \overline{\varphi_{k}(z)}-\varphi_{k}^{*}(z)\right] \overline{d z} \\
\beta_{k}=\left(N^{\prime}-1, \varphi_{k}^{\prime}\right)=-\frac{q}{4 \pi} \int_{\mathfrak{C}} \varphi_{k}^{*}(z)\left[\int_{\mathfrak{C}} \frac{t \overline{d t}}{(\bar{t}-\bar{z})^{2}}\right] \overline{d z} .
\end{gathered}
$$


Die Gleichheit in (7) gilt zunächst im Sinne der Normkonvergenz, dann aber auch im Sinne gleichmäßiger Konvergenz der rechts stehenden Reihen „im Innern“ bzw. sogar in $\overline{\mathfrak{G}}$. In (8) fasse man die äußere Integration bei $\beta_{k}$ über einen Grenzübergang mit einer äußeren Niveaulinie $\mathfrak{C}^{\prime}$ von $\mathfrak{C}$ auf (entsprechend $|\zeta|=r>1$ ). Für die unten benutzten $\varphi_{k}$ kann wegen Kelloggscher Sätze [16] die Begründung von (8) auch einfacher über die durch partielle Integration transformierten Integrale in (14) ff. von [7] erfolgen.

Analoge Darstellungen finden sich in [7] für diejenigen Funktionen $M(z, \zeta)$, $N(z, \zeta)$ mit endlichem ,Aufpunkt" $\zeta \in(\mathfrak{b}$, entstehend aus entsprechenden Funktionen $g_{\vartheta}(z, \zeta)$. Bei letzteren liege - bei sonst gleichen Eigenschaften -- der einfache Pol in $\zeta$ mit der Entwicklung

$$
g_{\vartheta}(z, \zeta)=\frac{1}{z-\zeta}+((0))
$$

\section{Ein spezielles Orthonormalsystem}

Es sei $\zeta(z)$ diejenige schlichte konforme Abbildung von $\mathfrak{G}$ auf $|\zeta|>1$ mit $\zeta(\infty)=\infty$. Wegen des konformen Radius 1 von $\mathfrak{G}$ können wir in Umgebung von $z=\infty$ die Normierung $\zeta(z)=z+\mathfrak{P}(1 / z)$ vorschreiben. Das System der ersten Ableitungen der Funktionen $F^{n}(z)=\zeta^{-n}(z), n=1,2, \ldots$, ist offenbar vollständig (da in der $\zeta$-Ebene trivialerweise der entsprechende Sachverhalt vorliegt) und soll jetzt orthonormiert werden. Das erheischt u. a. die Berechnung der zugehörigen Skalarprodukte.

Zunächst erkennen wir mit den Faberschen Polynomen $\Phi_{n}(z)$ (vgl. z. B. [13])

$$
\zeta^{n}=F^{-n}=\Phi_{n}(z)-n \sum_{l=1}^{\infty} a_{n l} \zeta^{-l} .
$$

Dabei sind $a_{n l}$ die „Grunsky-Koeffizienten” von $z(\zeta)$, die sich aus der Entwicklung

$$
\log \frac{z(\zeta)-z(\eta)}{\zeta-\eta}=-\sum_{k, l=1}^{\infty} a_{k l} \zeta^{-k} \eta^{-l}, \quad|\zeta|>1, \quad|\eta|>1,
$$

berechnen und Polynome in den Entwicklungskoeffizienten von $z(\zeta)$ selbst sind. Es gilt nun

$$
F^{n *}=q F^{-n}-q \Phi_{n}=-q n \sum_{l=1}^{\infty} a_{n l} \zeta^{-l} \text { für } \quad z \in \mathfrak{G} \text { bzw. }|\zeta|>1 .
$$

Denn diese Funktion ist noch in $z=\infty$ regulär und verschwindet dort, und es gilt auf $\mathfrak{C}$

$$
q \overline{F^{n}}-F^{n *}=q F^{-n}-F^{n *}=q \Phi_{n}(z),
$$

so daß dies ins Innere von $\mathfrak{C}$ analytisch fortsetzbar ist. 
Man errechnet weiter

$$
\begin{gathered}
\left(F^{m^{\prime}}, F^{n^{\prime}}\right)=-\frac{1}{2 i} \int_{\mathfrak{C}} F^{m} d \overline{F^{n}}+\overline{F^{m *}} d F^{n *} \\
=-\frac{1}{2 i} \int_{\mathfrak{C}}\left[F^{m} d F^{-n}+q^{2} \overline{\left(F^{-m}-\Phi_{m}\right)} d\left(F^{-n}-\Phi_{n}\right)\right] \\
=-\frac{1}{2 i} \int_{\mathfrak{C}}\left[-n\left(1+q^{2}\right) F^{m-n-1} d F-q^{2} F^{m} d \Phi_{n}-q^{2} \overline{\Phi_{i n} d F^{n}}+q^{2} \overline{\Phi_{m}} d \Phi_{n}\right], \\
\left(F^{m^{\prime}}, F^{n \prime}\right)=\left\{\begin{aligned}
n\left(1-q^{2}\right) \pi-\frac{q^{2}}{2 i} \int_{\mathfrak{C}} \overline{\Phi_{n}} d \Phi_{n} & \text { für } \quad m=n, \\
-\frac{q^{2}}{2 i} \int_{\mathfrak{C}} \overline{\Phi_{m}} d \Phi_{n} & \text { für } m \neq n .
\end{aligned}\right.
\end{gathered}
$$

Denn es ist

$$
\begin{gathered}
\int \Phi_{m} d F^{n}=\int \Phi_{m} d \zeta^{-n}=\int \Phi_{m}(z(\zeta))(-n) \zeta^{-n-1} d \zeta \\
=\int\left(\zeta^{m}+m \sum_{l=1}^{\infty} a_{m l} \zeta^{-l}\right)(-n) \zeta^{-n-1} d \zeta
\end{gathered}
$$

mit dem Ergebnis $n 2 \pi i$ für $m=n$ und 0 sonst.

Eine weitere, für andere Zwecke u.U. günstigere Darstellung des Skalarproduktes ergibt sich so nach (11)

$$
\begin{gathered}
\left(F^{m^{\prime}}, F^{n^{\prime}}\right)=-\frac{1}{2 i} \int_{\mathfrak{c}} F^{m} d \overline{F^{n}}+\overline{F^{m *}} d F^{n *} \\
=-\frac{1}{2 i} \int_{\mathfrak{C}}\left[\zeta^{-m} n \zeta^{n-1} d \zeta+q^{2} m n \sum_{k=1}^{\infty} \overline{a_{m k}} \zeta^{k} d \sum_{l=l}^{\infty} a_{n l} \zeta^{-l}\right] \\
=\left\{\begin{array}{lll}
\pi n-\pi q^{2} n^{2} \sum_{k=1}^{\infty} k \overline{a_{n k}} a_{n k} & \text { für } & m=n, \\
-\pi q^{2} m n \sum_{k=1}^{\infty} k \overline{a_{m k}} a_{n k} & \text { für } & m \neq n .
\end{array}\right.
\end{gathered}
$$

Falls $\mathfrak{C}$ nicht durchweg analytisch ist, geht man beim Beweis von (12) und der eben gewonnenen Darstellung bei dem bei der Definition von $\left(F^{m^{\prime}}, F^{n^{\prime}}\right)$ zugrunde liegenden Flächenintegral zunächst zu einem von einer Niveaulinie $\mathfrak{C}^{\prime}$ berandeten Teilgebiet von $\mathfrak{5}$ über (entsprechend $|\zeta|>r$ mit $r>1$ ) und führt anschließend den Grenzübergang $r \rightarrow 1$ durch. Dies liefert dann bei (12) das Ergebnis, bei der eben gewonnenen zweiten Darstellung nach dem Abelschen Grenzwertsatz, da die entstandene Reihe tatsächlich noch (für $r=1$ ) konvergiert, sogar absolut:

$$
\left(\sum_{k} k\left|a_{m k}\right|\left|a_{n k}\right|\right)^{2} \leqq \sum_{k} k\left|a_{m k}\right|^{2} \sum_{k} k\left|a_{n k}\right|^{2} \leqq 1 /(m n) .
$$

Die letzte Abschätzung ergibt sich im Zusammenhang mit den Grunskyschen Koeffizientenbedingungen ([13], (15) auf S. 59).

Setzt man noch

$$
C_{m n}=\sqrt{m n} a_{m n}, \quad D_{m n}=\sum_{k=1}^{\infty} \overline{C_{m k}} C_{n k},
$$


so entsteht die Matrix $\left(D_{m n}\right)$ aus den Matrizen $\left(\overline{C_{m n}}\right)$ und $\left(C_{m n}\right)$ durch Matrizenmultiplikation, und es folgt endgültig diese zweite Darstellung:

$$
\left(F^{m^{\prime}}, F^{n^{\prime}}\right)=\left\{\begin{array}{lll}
\pi n-\pi q^{2} n D_{n n} & \text { für } & m=n, \\
-\pi q^{2} \sqrt{m n} D_{m n} & \text { für } & m \neq n .
\end{array}\right.
$$

Die bekannte Orthonormierung nach E. Schmidt führt nun zu einem System von Funktionen

$$
\varphi_{n}=\sum_{k=1}^{n} \alpha_{k n} F^{k}, \quad n=1,2, \ldots,
$$

mit $\left(\varphi_{m}^{\prime}, \varphi_{n}^{\prime}\right)=\delta_{m n}$ (Kronecker-Symbol), wobei sich die Koeffizienten $\alpha_{k n}$ explizit durch Determinanten ausdrücken lassen (vgl. z. B. [2], S. 16/17). Es ist z. B.

$$
\alpha_{11}=\left(\pi-\pi q^{2} D_{11}\right)^{-1 / 2}, \quad \alpha_{1 n}=(-1)^{n} \Delta_{n}^{*}\left(\pi \Delta_{n}^{* *} \Delta_{n}\right)^{-1 / 2} \quad \text { für } \quad n>1
$$

mit

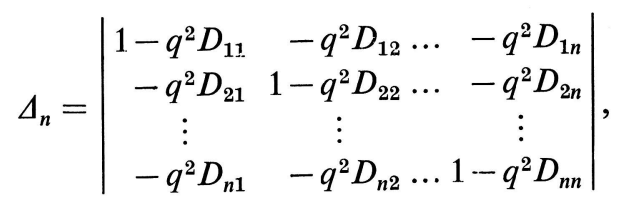

wobei $\Delta_{n}^{*}$ bzw. $\Delta_{n}^{* *}$ aus $\Delta_{n}$ durch Streichung von erster Zeile und letzter Spalte bzw. letzter Zeile und letzter Spalte entsteht.

Wir benötigen für $z \in \mathfrak{G}$ noch die Funktion

$$
F^{n * *}(z)=-q n\left(\sum_{l=1}^{\infty} a_{n l} \zeta^{-l}(z)\right)^{*}=-q^{2} n \frac{1}{2 \pi i} \int_{\mathbb{E}} \sum_{l=1}^{\infty}{\overline{a_{n l}}}_{\zeta}^{l}(t) \frac{d t}{t-z} .
$$

Hier lassen sich die Integrale umschreiten in solche über einen zu $z$ konzentrischen Kreis und solche über einen grossen Kreis $\mathfrak{R}$ nach Vertauschung von Summation und Integration und Berücksichtigung von [13] (dort (3) auf S. 57):

$$
\begin{gathered}
F^{n * *}(z)=-q^{2} n \sum_{l=1}^{\infty} \overline{a_{n l}}\left[\zeta^{l}(z)+\frac{1}{2 \pi i} \int_{\Omega} \zeta^{l} \frac{d t}{d \zeta} \frac{d \zeta}{t(\zeta)-z}\right] \\
=-q^{2} n \sum_{l=1}^{\infty} \overline{a_{n l}}\left[\zeta^{l}(z)+\frac{1}{2 \pi i} \int_{\Omega} \zeta^{l} \sum_{k=0}^{\infty} \Phi_{k}(z) \zeta^{-k-1} d \zeta\right] \\
=-q^{2} n \sum_{l=1}^{\infty} \overline{a_{n l}}\left[\zeta^{l}(z)-\Phi_{l}(z)\right]=q^{2} n \sum_{l=1}^{\infty} \overline{a_{n l}} l \sum_{k=1}^{\infty} a_{l k} \zeta^{-k}(z) .
\end{gathered}
$$

Falls $\mathfrak{C}$ nicht durchweg analytisch ist, geht man analog vor wie bei der Begründung von (15) mit dem Abelschen Grenzwertsatz. Dabei wird dann analog zu (13) die folgende Ungleichung benutzt, die nach [13] (dort (15) auf S. 59) für alle endlichen Wertesysteme $k, l$ gültig ist :

$$
\begin{aligned}
& \left(\sum_{k, l} l\left|a_{n l}\right|\left|a_{l k}\right||\zeta|^{-k}\right)^{2}=\left(\sum_{k, l} \sqrt{l}\left|a_{n l}\right||\zeta|^{-k / 2} \sqrt{l}\left|a_{l k}\right||\zeta|^{-k / 2}\right)^{2} \\
& \leqq \sum_{k, l} l\left|a_{n l}\right|^{2}|\zeta|^{-k} \sum_{k, l} l\left|a_{l k}\right|^{2}|\zeta|^{-k} \leqq \sum_{l} l\left|a_{n l}\right|^{2} \sum_{k}|\zeta|^{-k} \sum_{k}|\zeta|^{-k} / k \text {. }
\end{aligned}
$$


Dies zeigt die absolute Konvergenz der für $F^{n * *}$ entstandenen Reihe und Umordnungsmöglichkeit zu

$$
F^{n * *}(z)=q^{2} n \sum_{k=1}^{\infty} D_{n k} \zeta^{-k} / \sqrt{n k} .
$$

\section{Berechnung der Darstellungen für $M$ und $N$}

Die Berechnung der Koeffizienten $\alpha_{k}$ nach (8) liefert bei unserem jetzt vorliegenden speziellen Orthonormalsystem bei wieder entsprechender Behandlung der Reihen

$$
\begin{gathered}
\overline{\alpha_{n}}=\pi q \alpha_{1 n}+\frac{q}{2 i} \sum_{k=1}^{n} \alpha_{k n} k \int_{\mathfrak{C}} \overline{\left(-\sum_{l=1}^{\infty} a_{k l} \zeta^{-l}\right)} \frac{d z}{d \zeta} d \zeta \\
=\pi q \alpha_{1 n}-\frac{q}{2 i} \sum_{k=1}^{n} \alpha_{k n} k \int_{\mathfrak{c}} \sum_{l=1}^{\infty} \overline{a_{k l}} \zeta^{l}\left(1-\sum_{j=1}^{\infty} j a_{j 1} \zeta^{-j-1}\right) d \zeta \\
=\pi q \alpha_{1 n}-\pi q \sum_{k=1}^{n} \alpha_{k n} \sqrt{k} D_{k 1} .
\end{gathered}
$$

Zur Vereinfachung können wir noch ausnutzen, daß

$$
\begin{gathered}
\delta_{j n}=\left(\varphi_{j}^{\prime}, \varphi_{n}^{\prime}\right)=\sum_{k, l} \alpha_{k j} \overline{\alpha_{l n}}\left(F^{k^{\prime}}, F^{\prime \prime}\right) \\
=-\pi q^{2} \sum_{k, l} \alpha_{k j} \overline{\alpha_{l n}} \sqrt{k l} D_{k l}+\pi \sum_{k=1}^{\min (j, n)} k \alpha_{k j} \overline{\alpha_{k n}},
\end{gathered}
$$

was sukzessive für $j=1, \ldots, n-1$ bei $n>1 \mathrm{zu}$

$$
q^{2} \sqrt{j} \sum_{k=1}^{n} \alpha_{k n} \sqrt{k} D_{k j}=j \alpha_{j n}
$$

führt, nebst entsprechenden Relationen für die anschließenden Werte $j=n, n+1, \ldots$. So erhalten wir - (20) für $j=1$ ausnutzend, sowie (17) -

$$
\alpha_{n}=\left\{\begin{array}{rrr}
\frac{1}{q \overline{\alpha_{11}}}-\pi\left(\frac{1}{q}-q\right) \overline{\alpha_{11}} & \text { für } & n=1, \\
-\pi\left(\frac{1}{q}-q\right) \overline{\alpha_{1 n}} & \text { für } & n>1 .
\end{array}\right.
$$

Dadurch ist mit (17) die Berechnung von $M$ gelungen. Insbesondere ergibt sich für die Entwicklungskoeffizienten in (5)

$$
\frac{1}{2}\left(A_{1,0}-A_{1, \pi / 2}\right)=\frac{1}{q}-\pi\left(\frac{1}{q}-q\right) \sum_{n=1}^{\infty}\left|x_{1 n}\right|^{2} .
$$

Das Doppelte dieser Größe heißt „quasikonforme Spanne“ $S$. Wir erhalten aus (22) insbesondere mit dem Inhalt $I$ des Inneren von $\mathfrak{C}$ die Abschätzung

$$
\begin{aligned}
& S / 2 \leqq \frac{1}{q}-\pi\left(\frac{1}{q}-q\right)\left|\alpha_{11}\right|^{2}=\frac{1}{q}-\pi\left(\frac{1}{q}-q\right)\left[\pi\left(1-q^{2} D_{11}\right)\right]^{-1} \\
& =\frac{1}{q}-\left(\frac{1}{q}-q\right)\left[1-q^{2} \sum k\left|a_{1 k}\right|^{2}\right]^{-1}=q \frac{I}{\pi} /\left[1-q^{2}\left(1-\frac{I}{\pi}\right)\right] .
\end{aligned}
$$


Besitzt $\mathfrak{G}$ nicht notwendig wie hier den konformen Radius 1, sondern den konformen Radius $R$, folgt hieraus durch Ähnlichkeitstransformation

$$
S / 2 \leqq q \frac{I}{\pi} /\left[1-q^{2}\left(1-\frac{I}{\pi R^{2}}\right)\right] .
$$

Dies ist nach Bergman und Schiffer (vgl. S. 126 in [8]) schärfer als (6) (rechts) in [9].

Etwas komplizierter ist die Berechnung der $\beta_{k}$ nach (8). Wenn also dort bei der äußeren Integration statt $\mathfrak{C}$ zunächst eine Niveaulinie $\mathfrak{C}^{\prime}$ (entsprechend $|\zeta|=r>1$ ) verwendet wird, welche anschließend nach $\mathfrak{C}$ strebt, wird nach [13] (dort (3) auf S. 57)

$$
\begin{gathered}
\beta_{n}=\frac{q^{2}}{4 \pi} \int_{\mathfrak{C}} \sum_{k=1}^{n} \overline{\alpha_{k n}} k \sum_{l=1}^{\infty} a_{k l} \zeta^{-l}(z)\left[\int_{\mathbb{C}} \frac{t \overline{d t}}{(\bar{t}-\bar{z})^{2}}\right] \overline{d z} \\
=\frac{q^{2}}{4 \pi} \sum_{k=1}^{n} \overline{\alpha_{k n}} k \sum_{l=1}^{\infty} a_{k l} \int_{\mathfrak{C}} t \overline{\left[\int_{\mathfrak{C}} \zeta^{l} \frac{d z}{d \zeta}(t-z(\zeta))^{-2} d \zeta\right]} \overline{d t} \\
=\frac{q^{2}}{4 \pi} \sum_{k=1}^{n} \overline{\alpha_{k n}} k \sum_{l=1}^{\infty} a_{k l} \int_{\mathfrak{C}} t\left[2 \pi i \overline{\Phi_{l}^{\prime}(t)}\right] \overline{d t} .
\end{gathered}
$$

Hierbei gilt

$$
\int_{\mathfrak{C}} t \overline{\Phi_{l}^{\prime}(t)} \overline{d t}=\int_{\mathfrak{C}} \Phi_{1}(t) \overline{d \Phi_{l}(t)}
$$

so daß wir nach (12) zunächst erhalten

$$
\beta_{n}=\pi q^{2} \sum_{k=1}^{n} \overline{\alpha_{k n}} k\left[\sum_{l=1}^{\infty} a_{k l} v^{\prime} \bar{l} D_{l 1}-a_{k 1}\right] .
$$

Diese Reihe konvergiert tatsächlich (was wir wieder im Zusammenhang mit dem Abelschen Grenzwertsatze für den Grenzübergang $\mathfrak{C}^{\prime} \rightarrow \mathfrak{C}$ brauchen). Nach den Grunskyschen Koeffizientenbedingungen (vgl. [13], Aufgabe 6 auf S. 64) gilt nämlich zu festem $k$

$$
\begin{aligned}
\left|\sum_{l=r}^{s} C_{k l} D_{l 1}\right|^{2} & =\left|\sum_{l=r}^{s} C_{k l}\left(\sum_{j=1}^{\infty} \overline{C_{l j}} C_{1 j}\right)\right|^{2}=\left|\sum_{l=r}^{s} \sum_{j=1}^{\infty} C_{l j} \overline{C_{k l}} \overline{C_{1 j}}\right|^{2} \\
& \leqq \sum_{l=r}^{s}\left|C_{k l}\right|^{2} \sum_{j=1}^{\infty}\left|C_{1 j}\right|^{2} \leqq \sum_{l=r}^{s}\left|C_{k l}\right|^{2}
\end{aligned}
$$

Man kann auch noch in (24) durch Vertauschung der Summationsreihenfolge umformen $\mathrm{zu}$

$$
\beta_{n}=\pi q^{2} \sum_{k=1}^{n} \overline{\alpha_{k n}} \sqrt{k}\left[\sum_{j=1}^{\infty} \overline{D_{k j}} C_{1 j}-C_{k 1}\right]
$$

Dies ist möglich wegen

$$
\begin{gathered}
\left|\sum_{l=1}^{\infty} C_{k l} D_{l 1}-\sum_{j=1}^{s} \overline{D_{k j}} C_{1 j}\right|^{2} \\
=\left|\sum_{l=1}^{\infty} C_{k l}\left(\sum_{j=1}^{\infty} \overline{C_{l j}} C_{1 j}\right)-\sum_{j=1}^{s}\left(\sum_{l=1}^{\infty} C_{k l} \overline{C_{j l}}\right) C_{1 j}\right|^{2} \\
=\left|\sum_{l=1}^{\infty} C_{k l}\left(\sum_{j=s+1}^{\infty} \overline{C_{l j}} C_{1 j}\right)\right|^{2} \leqq \sum_{l=1}^{\infty}\left|C_{k l}\right|^{2} \sum_{l=1}^{\infty}\left|\sum_{j=s+1}^{\infty} C_{l j} \overline{C_{1 j}}\right|^{2} \leqq 1 \sum_{j=s+1}^{\infty}\left|C_{1 j}\right|^{2}
\end{gathered}
$$


Dabei werden abermals die Grunskyschen Koeffizientenbedingungen benutzt vgl. z. B. in [13] auf S. 60 unter (21).

Zur weiteren Vereinfachung von (26) kann man dann noch die Beziehungen (20) heranziehen.

Wir fassen zusammen.

Satz 1. Die in $\S 2$ definierten Grundlösungen $M(z)$ und $N(z)$ lassen sich in der Form (7) darstellen, wobei die Koeffizienten (8) bzw. (21), (24), (26) erfüllen. Die Gleichheit in (7) gilt im Sinne der Normkonvergenz und auch im Sinne gleichmäßiger Konvergenz auf jeder abgegeschlossenen Teilmenge von $\mathbf{6}$.

Diese Aussage läßt sich noch wie in $\S 5$ konkretisieren.

Zuvor erwähnen wir noch, wie sich entsprechend die in $\S 2$ genannten Funktionen $M(z, \zeta)$ und $N(z, \zeta)$ mit endlichem Aufpunkt $\zeta$ herstellen lassen. Es gilt nach [7] und (19) z. B.

$$
\begin{gathered}
M^{\prime}(z, 3)=\sum_{n}\left(M^{\prime}(Z, 3), \varphi_{n}^{\prime}(Z)\right) \varphi_{n}^{\prime}(z) \\
=\sum_{n} \sum_{k=1}^{n} \overline{\alpha_{k n}} \overline{\left(F^{k^{\prime}}(Z), M^{\prime}(Z, 3)\right)} \sum_{l=1}^{n} \alpha_{l n} F^{\prime \prime}(z) \\
=\sum_{n} \sum_{k, l=1}^{n} \overline{\alpha_{k n}} \alpha_{l n} \overline{\left[\pi q F^{k^{\prime}}(3)-\frac{\pi}{q} F^{k * *^{\prime}}(3)\right]} F^{l^{\prime}(z)} \\
=\sum_{n} \sum_{k, l=1}^{n} \overline{\alpha_{k n}} \alpha_{l n} \pi q \overline{\left[F^{k^{\prime}}(3)+\sum_{j=1}^{\infty} \sqrt{k j} D_{k j} \zeta^{-j-1} \frac{d \zeta}{d \mathfrak{Z}}\right]} F^{l^{\prime}(z) .}
\end{gathered}
$$

\section{Fehlerabschätzungen bei analytischer Kurve $\mathfrak{C}$}

a) Wir wollen nun in (7) den Fehler ermitteln, der entsteht, wenn nur endlich viele Summanden genommen werden. Dabei sei hier zunächst $\mathfrak{C}$ durchweg analytisch (d. h. ohne „Knicke“), so daß die Umkehrung der Riemannschen Abbildungsfunktion $\zeta(z)$ von $\mathfrak{G}$ noch für $|\zeta| \geqq \varrho$ regulär und schlicht ist mit einem $\varrho<1$. Diese Größe $\varrho$ spielt - wie immer in solcher Situation - naturgemäß jetzt eine zentrale Rolle. Als Funktion von $\zeta$ können wir dann $M$ nach $|\zeta| \geqq \varrho$ analytisch fortsetzen (vgl. die Schlußweise z. B. in [5], Fußnote). Wir entwickeln dort dieses $M^{*}$ :

$$
M^{*}=\sum_{v=1}^{\infty} \alpha_{v}^{*} \zeta^{-v} .
$$

Nach der Cauchyschen Abschätzungsformel können wir für

$$
\alpha_{v}^{*}=\frac{1}{2 \pi i} \int_{|\zeta|=\varrho} M^{*} \zeta^{v-1} d \zeta
$$

eine Ungleichung anschreiben, dabei mit benutzen, daß nach [5] die analytische 
Fortsetzung von $g_{0}$ bzw. $g_{\pi / 2}$ lautet

$$
\begin{aligned}
& g_{0}(z(\zeta))-q \overline{g_{0}(z(\zeta))}+q \overline{g_{0}(z(1 / \bar{\zeta}))} \text { bzw. } \\
& g_{\pi / 2}(z(\zeta))+q \overline{g_{\pi / 2}(z(\zeta))}-q \overline{g_{\pi / 2}(z(1 / \bar{\xi}))} .
\end{aligned}
$$

Also gilt für diese analytische Fortsetzung $M^{*}$ von $M$ nach $\varrho \leqq|\bar{\zeta}| \leqq 1$, da bei Integration in (30) die Anteile über die dritten Terme in (31) verschwinden für $v>1$,

$$
\left|\alpha_{v}^{*}\right| \leqq(1+q) K \varrho^{\nu} \text {, wenn }\left|g_{0}\right| \leqq K \quad \text { und } \quad\left|g_{\pi / 2}\right| \leqq K \quad \text { auf }|\zeta|=\varrho .
$$

Nun können wir zur Normabschätzung des Fehlers' bei $M^{\prime}$ schreiten. Zunächst folgt nach (4)

$$
\iint_{\mathfrak{G}}\left|M^{\prime}-\sum_{v=1}^{n} \alpha_{v} \varphi_{v}^{\prime}\right|^{2} d x d y \leqq \frac{1}{1-q^{2}}\left\|M^{\prime}-\sum_{v=1}^{n} \alpha_{v} \varphi_{v}^{\prime}\right\|^{2}=\frac{1}{1-q^{2}} \sum_{v=n+1}^{\infty}\left|\alpha_{v}\right|^{2} .
$$

(Strich' bezeichnet nach wie vor die Ableitung nach z.) Andererseits ergibt sich aus der klassischen Minimaleigenschaft der Fourierkoeffizienten $\alpha_{v}$ sowie wiederum aus (4)

$$
\begin{gathered}
\left\|M^{\prime}-\sum_{v=1}^{n} \alpha_{v} \varphi_{v}^{\prime}\right\|^{2} \leqq\left\|M^{\prime}-\left(\sum_{v=1}^{n} \alpha_{v}^{*} \zeta^{-v}\right)^{\prime}\right\|^{2} \leqq \iint_{\mathfrak{G}}\left|M^{\prime}-\left(\sum_{v=1}^{n} \alpha_{v}^{*} \zeta^{-v}\right)^{\prime}\right|^{2} d x d y \\
=\iint_{|\zeta|>1}\left|\frac{d}{d \zeta} \sum_{v=n+1}^{\infty} \alpha_{v}^{*} \zeta^{-v}\right|^{2} d \xi d \eta=\pi \sum_{v=n+1}^{\infty} v\left|\alpha_{v}^{*}\right|^{2} \quad(\zeta=\xi+i \eta) .
\end{gathered}
$$

Wegen $\sum_{v=n+1}^{\infty} v \varrho^{2 v}=\left(n+1-n \varrho^{2}\right)\left(1-\varrho^{2}\right)^{-2} \varrho^{2 n+2}$ erhalten wir somit

$$
\sum_{v=n+1}^{\infty}\left|\alpha_{v}\right|^{2} \leqq \pi(1+q)^{2} K^{2}\left(n+1-n \varrho^{2}\right)\left(1-\varrho^{2}\right)^{-2} \varrho^{2 n+2},
$$

$$
\iint_{\mathfrak{G}}\left|M^{\prime}-\sum_{v=1}^{n} \alpha_{v} \varphi_{v}^{\prime}\right|^{2} d x d y \leqq \pi \frac{(1+q)^{2}}{1-q^{2}} K^{2}\left(n+1-n \varrho^{2}\right)\left(1-\varrho^{2}\right)^{-2} \varrho^{2 n+2} .
$$

Eine zu (36) entsprechende Abschätzung können wir herbeiführen, wenn wir in der vorgetragenen Überlegung durchweg $M$ durch $N-z$ ersetzen. Schreiben wir nämlich jetzt für die analytische Fortsetzung $N^{*}$ von $N$ nach $|\zeta| \geqq \varrho$

$$
N^{*}-z=\sum_{v=1}^{\infty} \beta_{v}^{*} \zeta^{-v}
$$

so folgt jetzt analog zu (32), wenn noch die aus klassischen Verzerrungssätzen der konformen Abbildung auf $|\zeta|=\varrho$ folgende Ungleichung $|z(\zeta)| \leqq 2 \varrho$ benutzt wird:

$$
\left|\beta_{v}^{*}\right| \leqq[(1+q) K+2 \varrho] \varrho^{v} .
$$


Insgesamt erhalten wir dann analog zu (35), (36):

$$
\iint_{\mathfrak{G}}\left|N^{\prime}-1-\sum_{v=1}^{n} \beta_{v} \varphi_{v}^{\prime}\right|^{2} d x d y \leqq \pi \frac{[(1+q) K+2 \varrho]^{2}}{1-q^{2}}\left(n+1-n \varrho^{2}\right)\left(1-\varrho^{2}\right)^{-2} \varrho^{2 n+2} .
$$

Wir können wie folgt zusammenfassen.

Satz 2. Bei Berechnung von $M$ und $N$ über die Reihen (7) gilt für den Abbruchfehler im Mittel, falls die Kurve $\mathfrak{C}$ durchweg analytisch ist und die genannte $\varrho$-Bedingung erfüllt,

$$
\iint_{\mathfrak{G}}\left|M^{\prime}-\sum_{v=1}^{n} \alpha_{v} \varphi_{v}^{\prime}\right|^{2} d x d y \leqq K_{1} \varrho^{2 n}, \quad \iint_{\mathfrak{G}}\left|N^{\prime}-1-\sum_{v=1}^{n} \beta_{v} \varphi_{v}^{\prime}\right|^{2} d x d y \leqq K_{2} \varrho^{\prime 2 n} .
$$

Dabei ist $\varrho^{\prime}$ beliebig mit $\varrho<\varrho^{\prime}<1$ wählbar; $K_{1}, K_{2}$ sind nur von $\varrho, \varrho^{\prime}$ und $q$ abhängig und explizit angebbar.

In bekannter Weise kann man hieraus auf die gleichmäßige Konvergenz in jeder abgeschlossenen Teilmenge von $\mathfrak{G}$ schließen mit einer Fehlerabschätzung der Form $\leqq$ const $\cdot \varrho^{\prime n}$; vgl. z. B. (16) auf S. 67 in [12] oder noch besser dort (6) auf S. 44 (dort nach Transformation der Norm in die $\zeta$-Ebene mit Verwendung der Bergmanschen Kernfunktion für $|\zeta|>1$ ). Wir werden unten sogar - möglicherweise z. Tl. schlechtere - Abschätzungen gleicher Art in $\overline{\mathfrak{5}}$ gewinnen.

Auch kann man leicht den Abbruchfehler für die Reihe (22) des Residuums von $M$ in $z=\infty$ abschätzen mit (21) und (35), ebenfalls für die entsprechende Reihe

$$
\frac{1}{2}\left(A_{1,0}+A_{1, \pi / 2}\right)=\sum_{v=1}^{\infty} \beta_{v} \alpha_{1 v}
$$

des Residuums von $N$ :

$$
\left|\sum_{v=n+1}^{\infty} \beta_{v} \alpha_{1 v}\right|^{2} \leqq \sum_{v=n+1}^{\infty}\left|\beta_{v}\right|^{2} \sum_{v=n+1}^{\infty}\left|\alpha_{1 v}\right|^{2} .
$$

Hierbei wird rechts mit (39) bzw. mit dem zuvor gefundenen Fehler bei (22) weiter gearbeitet.

b) Für praktische Berechnungen ist es natürlich wichtig, in (35) usw. möglichst kleine Konstanten $K$ zu kennen. Sofort kann man als einen möglichen Wert nach Verzerrungssätzen bei den hydrodynamisch normierten Abbildungen der Klasse $\Sigma$ wählen $K=2$. Aber es lassen sich in sehr verschiedener Weise wesentlich kleinere Werte wählen, dann abhängig von $\varrho$ und $q$, wenn man Verzerrungssätze bei nach $\varrho<|\zeta|<1$ quasikonform fortsetzbaren Abbildungen der Klasse $\Sigma$ heranzieht. Betrachtet man nämlich $g_{0}$ und $g_{\pi / 2}$ als Funktion von $\zeta$ für $|\zeta|>\varrho$, dann liegen hier Abbildungen $w(\zeta)$ der Klasse $\Sigma$ vor, die nach $\varrho<|\zeta|<1$ noch $Q$-quasikonform fortsetzbar sind mit $Q=(1+q) /(1-q)$. In unserer Betrachtungsweise ist also ein 
Wert für $K$ durch sup $\mathfrak{R} w(\varrho)$ gegeben. Da dieses Supremum nach allgemeinen Prinzipien durch eine zur reellen Achse symmetrische Abbildung angenommen wird, kann man auch durch eine Joukowski-Abbildung übergehen zur Klasse der hydrodynamisch normierten konformen Abbildung des Äußeren der Ellipse mit der Brennachse $-2 \varrho \ldots 2 \varrho$ und der großen Halbachse $1+\varrho^{2}$, die nach dem vollen Inneren $Q$-quasikonform fortsetzbar sind. In dieser Abbildungsklasse ist das entsprechende Extremalproblem in [11] studiert worden. Da dies jedoch keine handlichen Formeln für $K$ liefert, geben wir noch folgende etwas gröbere Variante an. Wir sperren nämlich besagte Ellipse in einen Kreis des Radius $1+\varrho^{2}$ ein und betrachten die hydrodynamisch normierten konformen Abbildungen des Äußeren dieses Kreises mit $Q$-quasikonformer Abbildung ins Innere. Dann wird hierzu in [9] der Wertebereich des Bildes von $2 \varrho$ abgeschätzt (in [9] auch Hinweise auf weitere Abschätzungen). Dies führt zu

$$
K=2 \varrho+\frac{2}{\pi}(Q-1)\left(1+\varrho^{2}\right) \mathbf{E}\left[1+\mathfrak{I} \mathfrak{g}^{2}\left(\frac{\pi \mathbf{K}^{\prime}}{4 \mathbf{K}}\right)\right]\left[1+Q \mathfrak{T} \mathfrak{g}^{2}\left(\frac{\pi \mathbf{K}^{\prime}}{4 \mathbf{K}}\right)\right]^{-1}
$$

Hierbei werden die üblichen Bezeichnungen für die vollständigen elliptischen integrale 1 . und 2 . Gattung verwendet, wobei $2 \varrho /\left(1+\varrho^{2}\right)$ einzusetzen ist.

c) Wir zeigen nun die gleichmäßige Konvergenz unserer Reihen sogar in $\overline{\mathfrak{G}}$. Wir gehen aus von der Norm $1 \operatorname{der} \varphi_{n}^{\prime}$ und erhalten nach (4)

$$
\begin{gathered}
\pi \sum_{k=1}^{n} k\left|\alpha_{k n}\right|^{2}=\iint_{|\zeta|>1}\left|\frac{d}{d \zeta} \sum_{k=1}^{n} \alpha_{k n} \zeta^{-k}\right|^{2} d \xi d \eta=\iint_{\mathfrak{G}}\left|\varphi_{n}^{\prime}\right|^{2} d x d y \\
\leqq\left\|\varphi_{n}^{\prime}\right\|^{2} /\left(1-q^{2}\right)=1 /\left(1-q^{2}\right) .
\end{gathered}
$$

Nach einem klassischen Löwnerschen Verzerrungssatze zur Abschätzung von $|d z / d \zeta|$ in der Klasse $\Sigma$ (vgl. [13], S. 65), hier aber auf die für $|\zeta|>\varrho$ schlichte Abbildung $z(\zeta)$ transformiert, gibt dies für $z \in \overline{\mathfrak{G}}$

$$
\begin{aligned}
& \left|\varphi_{n}^{\prime}(z)\right|^{2}=\left|\sum_{k=1}^{n} \alpha_{k n} k \zeta^{-k-1} \frac{d \zeta}{d z}\right|^{2} \leqq \sum_{k=1}^{n} k\left|\alpha_{k n}\right|^{2} \sum_{k=1}^{n} k|\zeta|^{-2 k-2}\left|\frac{d \zeta}{d z}\right|^{2} \\
& \leqq \frac{1}{\pi\left(1-q^{2}\right)}|\zeta|^{-4}(n / 2)(n+1)\left(1-\varrho^{2}|\zeta|^{-2}\right)^{-2}=\frac{n(n+1)}{2 \pi\left(1-q^{2}\right)\left(|\zeta|^{2}-\varrho^{2}\right)^{2}} .
\end{aligned}
$$

Kombinieren wir nun (46) mit der aus (35) bzw. (39) folgenden Abschätzung für $\left|\alpha_{v}\right|$ bzw. $\left|\beta_{v}\right|$, erhalten wir schon den

Satz 3. Bei Berechnung von $M$ und $N$ über die Reihen (7) gilt für den $A b$ bruchfehler, falls die Kurve $\mathfrak{C}$ durchweg analytisch ist und die genannte @-Bedingung erfüllt, für alle $z \in \overline{\mathfrak{G}}$

$$
\left|M^{\prime}-\sum_{v=1}^{n} \alpha_{v} \varphi_{v}^{\prime}\right| \leqq K_{1}^{*} \varrho^{\prime n}, \quad\left|N^{\prime}-1-\sum_{v=1}^{n} \beta_{v} \varphi_{v}^{\prime}\right| \leqq K_{2}^{*} \varrho^{\prime n} .
$$


Dabei ist $\varrho^{\prime}$ beliebig mit $\varrho<\varrho^{\prime}<1$ wählbar; $K_{1}^{*}, K_{2}^{*}$ sind nur von $\varrho, \varrho^{\prime}$ und $q$ abhängig und explizit angebbar.

Auf den an sich auf der rechten Seite von (47) noch möglichen Faktor $|\zeta|^{-2}$ wurde verzichtet, da sich die Formel mit diesem Faktor sofort aus (47) mit dem Maximumprinzip wieder gewinnen läßt, da die linke Seite in $z=\infty$ von zweiter Ordnung verschwindet.

d) Bemerkung zur tätlichen Durchführung. Nach Satz 2 bzw. 3 kann man zu gegebenem $\varepsilon>0$ effektiv angeben, bei welcher Wahl von $n$ sicher der Fehler kleiner als $\varepsilon$ ist. Dies setzt natürlich voraus, daß in den Reihen (7) die Summanden, d.h. die $\alpha_{v}, \beta_{v}$ und $\varphi_{v}^{\prime}$ exakt berechnet werden können. Ist die Riemannsche Abbildungsfunktion $F$ mit ihren Entwicklungskoeffizienten in $z=\infty$ exakt bekannt, kann man die Grunsky-Koeffizienten $a_{k l}$ und die Faber-Polynome $\Phi_{n}$ exakt berechnen. Das Gleiche gilt dann weiter für die $D_{k l}$ nach (12) und (15), falls man die Integration rechts in (12) geschlossen ausführen kann. Dies geht sicher dann, wenn man $\bar{z}$ auf $\mathfrak{C}$ als geeignete Funktion von $z$ ausdrücken kann, wie z. B. bei einer Ellipse oder einer Cassinischen Kurve oder bei den (unter den nächsten Paragraphen fallenden) Polygonzügen $\mathfrak{C}$. Damit können auch exakt die $\alpha_{k n}$ und also die $\varphi_{n}^{\prime}$ berechnet werden, also nach (21) ebenfalls die $\alpha_{n}$, die $\beta_{n}$ nach (24) bzw. (26) jedenfalls mit beliebiger Genauigkeit (mit z. B. nach (25) abschätzbarem Fehler wegen $\left|C_{k l}\right| \leqq \varrho^{k+l}$ ).

In vielen Fällen (z. B. im Zusammenhang mit dem Extremalproblem der quasikonformen Abbildung, dessen Lösung $g_{0}$ ist) wird man sich vorzüglich für die Reihe in (22) interessieren, die in ihrem Rest wegen des Zusammenhanges (21) der Summanden mit den $\alpha_{n}$ entsprechend abschätzbar ist.

\section{Fehlerabschätzung bei stückweis analytischer Kurve $\mathfrak{C}$}

Es sei jetzt $\mathfrak{C}$ eine Jordankurve, die sich aus endlich vielen abgeschlossenen analytischen Bögen zusammensetzt, die unter von Null verschiedenen Winkeln in gewissen „Ecken“ $z_{k}$ zusammenstoßen. Es sei $\pi \alpha$ der kleinste der in den Ecken vorliegenden Außenwinkel. Wir wollen aufzeigen, wie man auch in diesem allgemeineren Falle zu Fehlerabschätzungen gelangt, wobei man nur einige Passagen im vorigen Paragraphen modifizieren muß, diese allerdings entscheidend. Wir begnügen uns mit der Herleitung der Normabschätzung des Fehlers.

Zunächst kann man eine Jordankurve $\mathfrak{c}$ zeichnen, die vollständig in $|\zeta| \leqq 1$ liegt, mit $|\zeta|=1$ genau die Punkte $\zeta_{k}=F\left(z_{k}\right)$ gemeinsam hat, in die $\zeta_{k}$ mit einseitiger, den Einheitskreis nicht berührender Tangente einläuft und zwischen je zwei aufeinander folgenden $\zeta_{k}$ sogar analytisch ist, so da $z=z(\zeta)$ ins Äußere von $\mathfrak{c}$ analytisch und schlicht fortsetzbar ist. Dies erkennt man unter Zuhülfenahme des Spiegelungsprinzips aus der Tatsache, daß eine in $\zeta_{k}$ von $|\zeta|>1$ her einlaufende analytische Kurve, die $|\zeta|=1$ nicht berührt, in eine in $z_{k}$ glatt einlaufende Kurve übergeht, 
die keinen der beiden dort zusammenstoßenden Teilbögen von $\mathfrak{c}$ berührt; man benutze hierbei Sätze vom Kellogg-Warschawskischen Typ, z. B. Theorem IX. 9 auf S. 366 in [16] (nach Anwendung einer Potenztransformation, die $\mathfrak{C}$ in Umgebung von $z_{k}$ in eine glatte Kurve überführt).

Wir können nun unsere Funktion $M$ nach dem Äußeren von $\mathfrak{c}$ analytisch fortsetzen $\mathrm{zu}$ einer Funktion $M^{*}$, die wieder über (31) gewinnbar ist, wobei auf $\mathfrak{c}$ noch Stetigkeit vorliegt. In der Darstellung (30) der Koeffizienten $\alpha_{v}^{*}$ der Entwicklung (29) können wir nun $\mathfrak{c}$ als Integrationsweg wählen, dabei für $v>1$ in (31) die jeweils letzten Anteile weglassen. Um zu einer brauchbaren Abschätzung für $\alpha_{v}^{*}$ nach genannter Integraldarstellung zu gelangen, muß jetzt ein größerer Aufwand getrieben werden.

Die Anteile von $\mathfrak{c}$, die innerhalb eines Kreises $|\zeta|=\varrho_{1}<1$ liegen, lassen sich wie in (32) durch const $\varrho_{1}^{v}$ abschätzen. Dies wird sich als von vernachlässigbarer Größenordnung erweisen $\mathrm{zu}$ den Anteilen, die durch Integration über den Rest von $\mathfrak{c}$, nämlich in Umgebung der $\zeta_{k}$ entstehen. Betrachten wir z. B. den Anteil, der bei Integration über die beiden in $\zeta_{k}$ einlaufenden Teilbögen $\mathfrak{c}_{k}$ von $\mathfrak{c}$ entsteht:

$$
\int_{\mathfrak{c}_{k}}\left[g_{0}-q \overline{g_{0}}\right] \zeta^{v-1} d \zeta-\int_{\mathfrak{c}_{k}}\left[g_{\pi / 2}-q \overline{g_{\pi / 2}}\right] \zeta^{\nu-1} d \zeta .
$$

Hierbei ist z. B. der erste Anteil zu $g_{0}$

$$
\begin{gathered}
\int_{\mathfrak{c}_{k}}\left[g_{0}-q \overline{g_{0}}\right] \zeta^{v-1} d \zeta=\int_{\mathfrak{c}_{k}}\left[g_{0}\left(z_{k}\right)-q \overline{g_{0}\left(z_{k}\right)}\right] \zeta^{\nu-1} d \zeta \\
+\int_{\mathfrak{c}_{k}}\left[g_{0}(z(\zeta))-g_{0}\left(z_{k}\right)\right] \zeta^{\nu-1} d \zeta-q \int_{\mathfrak{c}_{k}}\left[\overline{g_{0}(z(\zeta))}-\overline{g_{0}\left(z_{k}\right)}\right] \zeta^{\nu-1} d \zeta .
\end{gathered}
$$

Hier läßt sich rechts das erste Integral ausrechnen; dieses ist jedenfalls vom Betrag $\leqq$ const $\cdot \varrho_{1}^{v}$ mit $\varrho_{1}<1$. Beim zweiten und dritten Integral rechts in (49) benutzen wir die $Q$-Quasikonformität mit $Q=(1+q) /(1-q)$ in einer kreisförmigen Umgebung von $z_{k}$ :

$$
\left|g_{0}(z)-g_{0}\left(z_{k}\right)\right| \leqq \text { const }\left|z-z_{k}\right|^{1 / Q}
$$

mit einer über klassische Verzerrungssätze explizit beschaffbaren Konstanten (vgl. z. B. [10], S. 69). Nimmt man noch die sich aus Kelloggschen Sätzen (vgl.z. B. [16], S. 365) ergebende Ungleichung

$$
\left|z-z_{k}\right| \leqq \text { const }\left|\zeta-\zeta_{k}\right|^{\alpha}
$$

hinzu, so ergibt diese zusammen mit (50) für den zweiten Term in (49) rechts (der dritte ist entsprechend $\mathrm{zu}$ behandeln) bei Verwendung der üblichen Bezeichnung der Beta- bzw. Gammafunktion und deren asymptotischen Verhaltens (mit jeweils gewissen i. allg. wieder verschiedenen Konstanten):

$$
\begin{gathered}
\left|\int_{\mathfrak{c}_{k}}\left[g_{0}(z(\zeta))-g_{0}\left(z_{k}\right)\right] \zeta^{v-1} d \zeta\right| \leqq \text { const } \int_{\mathfrak{c}_{k}}\left|\zeta-\zeta_{k}\right|^{\alpha / Q}|\zeta|^{v-1}|d \zeta| \\
\leqq \mathrm{const} \int_{\varrho_{1}}^{1} r^{v-1}(1-r)^{\alpha / Q} d r \leqq \mathrm{const} B(v, 1+\alpha / Q) \\
=\mathrm{const} \Gamma(v) \Gamma(1+\alpha / Q) / \Gamma(v+1+\alpha / Q) \leqq \text { const } v^{-1-\alpha / Q}
\end{gathered}
$$


In der Zusammenfassung bringt dies analog zu (32)

$$
\left|\alpha_{v}^{*}\right| \leqq \text { const } v^{-1-\alpha / Q} \text {. }
$$

Nach (33) und (34) fließt daraus jetzt wegen

$$
\sum_{v=n+1}^{\infty} v\left|\alpha_{v}^{*}\right|^{2} \leqq \text { const } n^{-2 \alpha / Q}
$$

Satz 4. Bei Berechnung von $M$ und $N$ über die Reihen (7) gilt für den Abbruchfehler im Mittel, falls die Kurve $\mathfrak{C}$ stückweis analytisch ist mit Außenwinkeln $\geqq \pi \alpha$ an den Eckpunkten,

$$
\iint_{\mathfrak{G}}\left|M^{\prime}-\sum_{v=1}^{n} \alpha_{v} \varphi_{v}^{\prime}\right|^{2} d x d y \leqq \text { const } n^{-2 \alpha / Q},
$$

nebst einer analogen Abschätzung mit $N^{\prime}-1$. Dabei ist, ,const“" nicht von $n$ abhängig und explizit angebbar, falls eine Kurve c bekannt ist.

Wie im Anschluß an Satz 2 kann man hieraus wieder unmittelbar auf die gleichmäßige Konvergenz in jeder abgeschlossenen Teilmenge von $(5$ schließen mit entsprechender Abschätzung.

\section{Beispiel: Ellipse}

Betrachten wir nun als (freilich extrem einfaches) Beispiel den Fall der Ellipse C, die durch

$$
z=z(\zeta)=\zeta+r^{2} / \zeta, \quad 0<r<1,
$$

aus $|\zeta|=1$ entsteht. Hier wird

$$
\begin{gathered}
a_{k k}=r^{2 k} / k, \quad a_{k l}=0 \text { für } k \neq l, \quad C_{k k}=r^{2 k}, \quad C_{k l}=0 \text { für } k \neq l, \\
D_{m m}=r^{4 m}, \quad D_{m n}=0 \text { für } m \neq n, \quad \alpha_{m m}^{-2}=\left(F^{m^{\prime}}, F^{m \prime}\right)=\pi\left(1-q^{2} r^{4 m}\right), \\
\alpha_{m n}^{-2}=\left(F^{m^{\prime}}, F^{n^{\prime}}\right)=0 \text { für } m \neq n,
\end{gathered}
$$

so daß die $F^{n}$ schon orthogonal sind. Es wird

$$
\varphi_{m}(z)=\left[\pi\left(1-q^{2} r^{4 m}\right)\right]^{-1 / 2} \zeta^{-m} .
$$

Wegen

$$
\begin{gathered}
\alpha_{1}=\pi q\left(1-r^{4}\right)\left[\pi\left(1-q^{2} r^{4}\right)\right]^{-1 / 2}, \quad \alpha_{k}=0 \quad \text { sonst, } \\
\beta_{1}=-\pi q^{2}\left(1-r^{4}\right) r^{2}\left[\pi\left(1-q^{2} r^{4}\right)\right]^{-1 / 2}, \quad \beta_{k}=0 \quad \text { sonst, }
\end{gathered}
$$

wird

$$
\begin{gathered}
M^{\prime}(z)=\pi q\left(1-r^{4}\right)\left[\pi\left(1-q^{2} r^{4}\right)\right]^{-1} \zeta^{-1}, \\
N^{\prime}(z)=1-\pi q^{2} r^{2}\left(1-r^{4}\right)\left[\pi\left(1-q^{2} r^{4}\right)\right]^{-1} \zeta^{-1},
\end{gathered}
$$


insbesondere nach (22) usw.

$$
\begin{gathered}
\frac{1}{2}\left(A_{1,0}-A_{1, \pi / 2}\right)=q\left(1-r^{4}\right) /\left(1-q^{2} r^{4}\right), \\
\frac{1}{2}\left(A_{1,0}+A_{1, \pi / 2}\right)=-q^{2} r^{2}\left(1-r^{4}\right) /\left(1-q^{2} r^{4}\right)
\end{gathered}
$$

in Übereinstimmung mit [4], wo diese Größen unter Zusatzbemerkung 1 im Zusammenhange mit einem Extremalproblem bei quasikonformen Abbildungen auftraten. Fehlerabschätzungen im Sinne von $\S 5$ sind in diesem Beispiel natürlich gegenstandslos.

Man kann so entsprechend die Funktionen $M$ und $N$ mit endlichem Aufpunkt (vgl. (9)) bestimmen. Dann entstehen statt (58), (59) tatsächlich unendliche Reihen. Dankbarere und aussagekräftigere Beispiele liegen vor, wenn $\mathfrak{C}$ z. B. eine Quadratlinie oder eine Cassinische Kurve ist.

\section{Verbesserung der Fehlerabschätzung mit Hilfe des Fredholmschen Eigenwertes}

Durch das Auftreten des Nenners $1-q^{2}$ werden obige Fehlerabschätzungen für $q$ nahe 1 immer schlechter. Dieses Übel läßt sich beheben, wenn von $\mathfrak{C}$ der kleinste Fredholmsche Eigenwert $\lambda$ (vgl. z. B. [14]) oder wenigstens eine Abschäızung für denselben bekannt ist. (Bei unseren Voraussetzungen an $\mathfrak{C}$ ist stets $\lambda>1$.) Der Grund besteht in folgender Verschärfung von (4).

Satz 5. Für die Funktionen $G^{\prime} \in \mathfrak{H}$ gilt sogar

$$
\left(1-q^{2} \lambda^{-2}\right) \iint_{\mathfrak{G}}\left|G^{\prime}\right|^{2} d x d y \leqq\left\|G^{\prime}\right\|^{2} \leqq \iint_{\mathfrak{F}}\left|G^{\prime}\right|^{2} d x d y .
$$

Der Faktor $1-q^{2} \lambda^{-2}$ ist bei gegebener Kurve $\mathfrak{C}$ nicht für alle $G^{\prime} \in \mathfrak{H}$ zu vergrößern.

Zwecks Beweis entwickeln wir ein beliebiges solches $G^{\prime}$ in der Form

$$
G(z)=\sum_{n=1}^{\infty} g_{n} F^{n}(z) \text { mit } \pi \sum_{n=1}^{\infty} n\left|g_{n}\right|^{2}=\iint_{\mathfrak{G}}\left|G^{\prime}\right|^{2} d x d y<\infty
$$

nach dem vollständigen System der $F^{n}$ von $\S 3$. Es wird hierzu nach (15)

$$
\begin{aligned}
\left\|G^{\prime}\right\|^{2} & =\pi \sum_{n=1}^{\infty} n\left|g_{n}\right|^{2}-\pi q^{2} \sum_{m, n=1}^{\infty} g_{m} \overline{g_{n}} m n\left(\sum_{k=1}^{\infty} k \overline{a_{m k}} a_{n k}\right) \\
& =\pi \sum_{n=1}^{\infty} n\left|g_{n}\right|^{2}-\pi q^{2} \sum_{k=1}^{\infty} k\left|\sum_{m=1}^{\infty} a_{m k} m \overline{g_{m}}\right|^{2},
\end{aligned}
$$

wobei - was schon (60) liefert - nach den verschärften Grunskyschen Koeffizientenbedingungen [8], [15] gilt

$$
\sum_{k=1}^{\infty} k\left|\sum_{m=1}^{\infty} a_{m k} m \overline{g_{m}}\right|^{2} \leqq \lambda^{-2} \sum_{m=1}^{\infty} m\left|g_{m}\right|^{2} .
$$

Nach [8] ist hier nicht für alle Systeme $g_{m}$ der Faktor $\lambda^{-2} \mathrm{zu}$ verkleinern. 
Wir bekommen nun aus Satz 5, wenn wir in $\S 5$ mit (60) statt mit (4) arbeiien, unmittelbar die

Folgerung. In (33), (36), (40), (45), (46) kann man den Nenner $1-q^{2}$ durch $1-q^{2} \lambda^{-2}$ ersetzen, wegen $\lambda \geqq \varrho^{-2}$ (vgl. [14]) auch durch $1-q^{2} \varrho^{4}$.

Somit sind $K_{1}, K_{2}$ in (41) und $K_{1}^{*}, K_{2}^{*}$ in (47) auch von $q$ unabhängig wählbar. Analoge Auswirkungen ergeben sich in $\S 6$.

Durch diesen Umstand erhält man überdies in den Darstellungen für $M$ und $N$ Reihen, die sogar für $q<\lambda$ konvergieren. Dies überrascht nicht nach [6], wo diese Funktionen tatsächlich für $q<\lambda$ betrachtet wurden.

Man kann übrigens Ungleichung (60) auch in der Form

$$
\iint_{\mathfrak{G}}\left|G^{* \prime}\right|^{2} d x d y \leqq q^{2} \lambda^{-2} \iint_{\mathfrak{G}}\left|G^{\prime}\right|^{2} d x d y
$$

schreiben. Dies gibt zusammen mit (6) in [7] Anlaß zu folgender Variationscharakterisierung von $\mathfrak{C}$.

Satz 6. Für alle Funktionen $G^{*}$, die in $(\mathfrak{5}$ (incl. $\infty$ ) regulär sind, ebenfalls im Komplement $\mathfrak{G}_{c}$ von $\mathfrak{G}$, wobei auf $\mathfrak{C}$ von beiden Seiten her stetige Randwerte existieren, deren konjugiert komplexe Differenz auf $\mathfrak{C}$ hölderstetig ist und die die stetigen Randwerte einer in $\mathbf{5}$ (incl. $\infty$ ) regulären Funktion darstellt, gilt

$$
\left(\lambda^{2}-1\right) \iint_{\mathfrak{G}}\left|G^{* \prime}\right|^{2} d x d y \leqq \iint_{\mathfrak{F}_{c}}\left|G^{* \prime}\right|^{2} d x d y .
$$

Der Faktor $\lambda^{2}-1$ kann nicht für alle derartigen $G^{*}$ vergrößert werden.

Denn die beschriebenen $G^{*}$ entstehen offenbar durch entsprechende Cauchyintegrale (vgl. (4) in [7]), so daß (63) anwendbar ist. Ferner lassen sich die in (63) zulässigen $G^{*}$ durch die in Satz 6 figurierenden (mit angegebenen Randwerten) entsprechend approximieren.

Wir bemerken noch abschließend, daß man auch die komplexen Eigenfunktionen im Sinne von Satz 5 in [6] statt des in $\S 3$ ff. zugrunde liegenden Systems der Funktionen $F^{n}$ zum Aufbau eines Orthonormalsystems verwenden kann, wieder mit dem Ziel einer Darstellung für $M$ und $N$. (Solche Darstellungen sind mehr von theoretischem Interesse, da diese Eigenfunktionen natürlich praktisch so schwer zu bestimmen sind wie $M$ und $N$ selbst; im Reellen finden sich analoge solche Darstellungen in [14], S. 1199 ff.). Auch bei diesen Eigenfunktionen kann man nämlich die *-Operation gemäß (2) explizit durchführen, wie übrigens auch, wenn man vom System der $M^{(k)}$ zu einem anderen $q$-Wert ausgeht in [7]. Interessant ist natürlich auch eine Darstellung der komplexen Eigenfunktionen durch Reihen nach den $F^{n}$, wie sie aus [15] folgt. 


\title{
Literatur
}

[1] Bergman, S., and M. Schiffer: Kernel functions and elliptic differential equations in mathematical physics. - Academic Press Inc., Publishers, New York, N. Y., 1953.

[2] GAIER, D.: Vorlesungen über Approximation im Komplexen. - Birkhäuser, Basel—BostonStuttgart, 1980.

[3] HoY; E.: Darstellungsformeln für gewisse quasikonforme Normalabbildungen. - Wiss. Z. Martin - Luther - Univ. Halle-Wittenberg Math.-Natur. Reihe (im Druck).

[4] KÜнNAU, R.: Bemerkungen zu den Grunskyschen Gebieten. - Math. Nachr. 44, 1970, 285293.

[5] KüHNAU, R.: Zur Methode der Randintegration bei quasikonformen Abbildungen. - Ann. Polon. Math. 31, 1976, 269-289.

[6] KüHNAU, R.: Eine Integralgleichung in der Theorie der quasikonformen Abbildungen. - Math. Nachr. 76, 1977, 139-152.

[7] KüHNaU, R.: Eine Kernfunktion zur Konstruktion gewisser quasikonformer Normalabbildungen. - Math. Nachr. 95, 1980, 229-235.

[8] KüHnAU, R.: Zu den Grunskyschen Koeffizientenbedingungen. - Ann. Acad. Sci. Fenn. Ser. A I Math. 6, 1981, 125-130.

[9] KüHNAU, R., und E. HoY: Bemerkungen über quasikonform fortsetzbare schlichte konforme Abbildungen. - Wiss. Z. Martin - Luther - Univ. Halle-Wittenberg Math.-Natur. Reihe 31, 1982, 129-133.

[10] Lehto, O., und K. I. VirTanen: Quasikonforme Abbildungen. - Springer-Verlag, Berlin-Heidelberg-New York, 1965.

[11] Maskus, R.: Ein Wertannahmeproblem bei quasikonform fortsetzbaren schlichten konformen Abbildungen des Äußeren einer Ellipse. - Rev. Roumaine Math. Pures Appl. (im Druck).

[12] Meschkowski, H.: Hilbertsche Räume mit Kernfunktion. - Springer-Verlag, Berlin-Göttingen-Heidelberg, 1962.

[13] Pommerenke, Chr.: Univalent functions. - Vandenhoeck \& Ruprecht, Göttingen, 1975.

[14] SCHIfFer, M.: The Fredholm eigen values of plane domains. - Pacific J. Math. 7, 1957, 11871225.

[15] Schiffer, M.: Fredholm eigenvalues and Grunsky matrices. - Ann. Polon. Math. 39, 1981, 149-164.

[16] TsusI, M.: Potential theory in modern function theory. - Maruzen Co., Ltd., Tokyo, 1959.

\author{
Martin-Luther-Universität Halle-Wittenberg \\ Sektion Mathematik \\ DDR-401 Halle an der Saale \\ Deutsche Demokratische Republik
}

Eingegangen am 2. Dezember 1983 